\title{
Maladies infectieuses (1)
}

\section{Infectious diseases (1)}

C SRLF et Springer-Verlag France 2011 tendait à être plus élevée chez les patients porteurs de Candida $s p$. que chez les autres $(5,43 \mathrm{vs} 3,11 \mathrm{ng} / \mathrm{ml} ; p=0,09)$.

Conclusion : La colonisation des voies aériennes par Candida $s p$. A été évaluée prospectivement dans une cohorte de patients suspects de PAVM. Elle apparaît comme un phénomène fréquent dans ce contexte, ce d'autant que $P$. aeruginosa est isolé dans les prélèvements respiratoires. Dans notre étude, cette colonisation fongique est un facteur de risque indépendant de mortalité en réanimation, comme précédemment rapporté. Ces résultats suggèrent un effet délétère de Candida sp. Sur l'évolution des PAVM.

\section{Références}

1. Roux D, Gaudry S, Dreyfuss D, et al (2009) Candida albicans impairs macrophage function and facilitates Pseudomonas aeruginosa pneumonia in rat. Crit Care Med 37:1062-7

2. Azoulay E, Timsit JF, Tafflet M, et al (2006) Candida colonization of the respiratory tract and subsequent pseudomonas ventilatorassociated pneumonia. Chest 129:110-7

\section{SP097 \\ Immunodépression et réanimation : émergence des maladies systémiques \\ T. Saghi ${ }^{1}$, A. Boyer ${ }^{2}$, M. Grenouillet-Delacre ${ }^{2}$, J. Pillot ${ }^{1}$, M. Saint-Léger ${ }^{3}$, B. Clouzeau ${ }^{2}$, D. Gruson ${ }^{2}$ \\ ${ }^{1}$ Réanimation, CHU Pellegrin-Tripode, Bordeaux, France \\ ${ }^{2}$ Service de réanimation médicale et de médecine hyperbare, CHU Pellegrin-Tripode, Bordeaux, France \\ ${ }^{3}$ Réanimation, hôpital Pellegrin-Tripode, Bordeaux, France}

Objectif : Préciser le pronostic et évaluer les caractéristiques démographiques, cliniques et biologiques des patients immunodéprimés hospitalisés en réanimation.

Patients et méthodes : À partir de janvier 2007, les caractéristiques de tout patient adulte immunodéprimé admis en réanimation ont été colligées. L'immunodépression était définie par : présence d'une hémopathie maligne ou un cancer « actif » ou en cours de traitement ou une leucopénie ou VIH positif ou un traitement par biothérapie ou un traitement au long cours (plus de trois mois) par corticoïde ou une greffe solide ou de cellules souches. La définition ne comportait pas les patients diabétiques, cirrhotiques ou insuffisants respiratoires chroniques sous corticothérapie au long cours, qui ont été exclus de l'analyse. Il s'agit d'une analyse monocentrique rétrospective épidémiologique, entrant dans le cadre de l'observation des infections nosocomiales, notamment chez les patients immunodéprimés.

Résultats : L'analyse a été faite suite à un recueil prospectif de 42 mois : 2071 patients ontété admis en réanimation, dont 226 patients immunodéprimés (soit $11 \%$ ) : âge moyen 58 ans ; IGS II moyen : 59 ; durée moyenne de séjour : 8,5 jours (dont $20 \% \geq 14$ jours). L'immunodépression principale était l'hémopathie maligne : 104 patients (46\%). Pour un tiers d'entre eux, il s'agissait d'une complication de traitement de leucémie aiguë, un tiers un lymphome 
et un tiers une complication précoce de greffe. L'incidence des autres immunodépressions était : patients VIH : 24 (11\%), greffes solides : 21 patients, cancer actif 14 patients et maladie systémique (MS) : 63 patients $(28 \%)$. Au moins deux défaillances d'organes étaient présentes pour $82 \%$ des cas. Les connectivites représentaient la majorité des MS. Les «portes » d'entrée en réanimation des MS étaient une défaillance pulmonaire souvent associée à une insuffisance rénale. Dans $68 \%$ des cas, une suspicion d'infection existait à l'entrée, avec une atteinte pulmonaire dans près de huit cas sur dix. Les patients avec insuffisance respiratoire aiguë fébrile ont bénéficié d'une VNI dans $35 \%$ des cas d'oncohématologie et $38 \%$ des cas de MS. Une ventilation mécanique a été nécessaire chez $60 \%$ des patients avec MS. Parmi les 154 patients suspects d'infection, 98 (64\%) ont eu une documentation microbiologique, dont $80 \%$ de bactéries. Parmi elles, $60 \%$ étaient des entérobactéries. La documentation microbiologique est à peine plus faible pour les MS que les hémopathies, en revanche $91 \%$ des micro-organismes dans les cas de MS étaient bactériens. Le taux de décès en réanimation des patients avec MS était de 36 versus $43 \%$ pour les patients d'oncohématologie.

Conclusion : Si dans notre unité, la cause première d'admission d'immunodépression reste les patients d'hématologie, l'incidence des patients avec maladie systémique sous de nouvelles biothérapies n'est pas négligeable. L'incidence des patients VIH semble avoir nettement diminué. Notre analyse révèle que les motifs d'admission sont infectieux, qu'ils sont largement documentés et bactériens dans près de neuf cas sur dix, à majorité bacilles à Gram négatif. Ce message semble important dans le choix probabiliste du traitement antimicrobien.

\section{SP098}

\section{Analyse rétrospective sur six années des syndromes d'activation lymphohystiocytaire secondaires à une infection survenus dans un service de réanimation polyvalente adulte \\ Y. Asencio ${ }^{1}$, G. Lacroix ${ }^{1}$, J. Bordes ${ }^{1}$, C. Martinaud ${ }^{2}$, E. Meaudre ${ }^{1}$ \\ ${ }^{1}$ Département d'anesthésie et réanimation, HIA Sainte-Anne, Toulon, \\ France \\ ${ }^{2}$ Département de biologie, HIA Sainte-Anne, Toulon, France}

Introduction : Les syndromes d'activation lymphohystiocytaire d'origine infectieuse (SALHI) correspondent à une stimulation exagérée des macrophages par les lymphocytes $\mathrm{T}$, réactionnelle à un agent infectieux souvent viral. Fièvre, hémophagocytose médullaire, cytopénies sanguines sur au moins deux lignées, hyperferritinémie, hypertryglycéridémie, hypofibrinogénémie et splénomégalie en sont les critères diagnostiques principaux proposés par Henter et al. [1]. Les SALHI sont rares, peu décrits en réanimation adulte et leur traitement est encore mal codifié [2].

Patients et méthodes : Étude rétrospective monocentrique du $1^{\text {er }}$ janvier 2004 au $1^{\text {er }}$ juillet 2010 dans un service de réanimation polyvalente adulte. Les dossiers étaient choisis sur la revue de tous les myélogrammes comportant des images d'hémophagocytose. Les dossiers comportant cinq critères diagnostiques proposés par Henter et al. [1] étaient sélectionnés. Les patients aux antécédents de cancers ou de connectivites étaient exclus. Les caractéristiques du séjour, les paramètres biologiques et cliniques le jour du diagnostic de SALHI, les agents infectieux et les modalités thérapeutiques étaient analysés. Les outils statistiques étaient le test de Wilcoxon et le test exact de Fischer. Le seuil de significativité retenu était $p<0,05$.

Résultats : Vingt patients ont présenté un SALHI, soit une incidence de $0,8 \%$ des admissions. Onze patients (55\%) étaient décédés. Les principaux agents infectieux associés à ces SALHI étaient une réactivation d'un cytomégalovirus dans $30 \%$ des cas, une infection profonde à Escherichia coli ou à Candida respectivement dans 25 et $10 \%$ des cas, une infection par le VIH dans $10 \%$ des cas. Dans $75 \%$ des cas, la période de survenue des SAHLI était postérieure d'une semaine à l'admission. Parmi les 19 patients traités par immunoglobulines humaines polyvalentes intraveineuses (IgIV), 13 (65\%) avaient une réponse thérapeutique favorable sans récidive (Tableau 1).

\begin{tabular}{|c|c|c|c|}
\hline Critères & $\begin{array}{l}\text { Survivants, } \\
n=11\end{array}$ & $\begin{array}{l}\text { Décédés, } \\
n=9\end{array}$ & $p$ \\
\hline Score IGS II & $35[26-52]$ & $52[40-89]^{*}$ & 0,04 \\
\hline $\begin{array}{l}\text { Survenue du SALHI } \\
\text { (jours) }\end{array}$ & $30[7-48]$ & $28[9-78]$ & 0,9 \\
\hline Plaquettes $\left(\times 10^{9} / 1\right)$ & 47 [40-69] & 50 [19-67] & 0,6 \\
\hline Hémoglobinémie (g/dl) & $8,6[6,8-11]$ & $8,7[8,4-9,6]$ & 0,7 \\
\hline LDH (UI/l) & $365[308-1235]$ & $222[134-279]^{*}$ & 0,005 \\
\hline Lactatémie $(\mathrm{mmol} / \mathrm{l})$ & $1,7[1,4-2,1]$ & $2,5[2,3-3,7]^{*}$ & 0,04 \\
\hline État de choc septique, $n(\%)$ & $7(63)$ & $4(44)$ & 0,33 \\
\hline $\begin{array}{l}\text { Traitement IgIV } \\
\text { efficace, } n(\%)\end{array}$ & $9(81)^{*}$ & $4(44)$ & 0,049 \\
\hline
\end{tabular}

Conclusion : Dans cette série, les agents infectieux réactionnels des SAHLI sont typiques [2]. La majorité des SAHLI sont acquis pendant le séjour en réanimation, à distance de l'admission. Le traitement par IgIV, conjointement à l'utilisation des anti-infectieux et du traitement des défaillances est, dans cette série, significativement associé à la survie des patients.

\section{Références}

1. Henter JI, Horne A, Aricó M, et al (2007) HLH-2004: Diagnostic and therapeutic guidelines for hemophagocytic lymphohistiocytosis. Pediatr Blood Cancer 48:124-31

2. Gonzalez F, Vincent F, Cohen Y (2009) Syndrome d'activation macrophagique d'origine infectieuse: étiologies et prise en charge. Réanimation 18:284-90

\section{SP099}

\section{Inhibiteur de la pompe à proton et pneumopathie chez les patients ventilés}

T. Vaysse ${ }^{1}$, G. Gouffran ${ }^{1}$, L. Lecuyer ${ }^{1}$, Y. Abadie $^{1}$, A. Van de Louw ${ }^{1}$, E. Brocas ${ }^{1}$, R. Boiteau ${ }^{1}$

${ }^{1}$ Service de réanimation polyvalente, centre hospitalier sud-francilien, site d'Évry, Paris, France

Introduction : Chez les patients traités par ventilation mécanique, le risque accru de pneumopathie nosocomiale lié à la prescription préventive d'inhibiteurs de la pompe à proton (IPP) reste débattu. Le but de cette étude est de vérifier si l'utilisation d'IPP à visée préventive chez les patients de réanimation, ventilés de manière invasive, augmente le risque de pneumopathie nosocomiale.

Patients et méthodes : Étude rétrospective analysant l'ensemble des patients admis dans un service de réanimation polyvalente et surveillance continue de 16 lits entre janvier 2003 et juillet 2010 et nécessitant une durée de ventilation mécanique invasive supérieure à 48 heures, après exclusion des patients admis pour une hémorragie digestive. Dans ce service de réanimation, la prescription préventive 
d'IPP (ésoméprazole ou oméprazole) n'est pas gérée par un protocole et est laissée au libre choix du prescripteur ; certains médecins de l'équipe sont favorables à ce traitement, d'autres y sont opposés ; toutefois, un traitement préventif décidé par un prescripteur à l'admission du patient n'est jamais interrompu. Huit cent cinquante et un patients ont été admis, et leurs données ont été enregistrées dans la base Clinisoft (GE). Les principaux motifs d'hospitalisation étaient une insuffisance respiratoire aiguë $(n=325, \mathrm{M}=38 \%)$, un coma $(n=192$, $\mathrm{M}=23,7 \%)$, un état de choc $(n=127, \mathrm{M}=14,9 \%)$.

Résultats : Cinq cent vingt patients $(61 \%)$ ont été traités par inhibiteur de la pompe à proton à une dose moyenne de $33,6 \mathrm{mg} / \mathrm{j}$ et 331 patients n'ont pas reçu de prévention antiulcéreuse. Il n'existe pas de différence significative de l'IGS II entre ces deux groupes $(58,06$ vs 57,32$)$. Il existe une réduction significative de l'incidence des pneumopathies nosocomiales dans le groupe sans prévention antiulcéreuse $(26,9 \% ; n=140)$ contre le groupe avec IPP $(15 \% ; n=50)[p<0,01]$. Le taux d'hémoglobine à l'entrée est plus faible dans le groupe avec IPP $(10,8$ vs $11,7 \mathrm{~g} / \mathrm{dl} ; p<0,01)$. L'étude montre aussi de manière significative $(p<0,01)$ une durée de séjour en réanimation $(9,7$ vs $8,21$ jours $; p<0,01)$ ainsi qu'une durée de ventilation mécanique $(17,93$ vs 13,37 jours $; p<0,01)$ plus longue dans le groupe avec IPP. Ces différences sont retrouvées dans les trois principaux groupes de motifs d'admission (insuffisance respiratoire aiguë, coma, choc) analysés séparément.

Conclusion : Ce travail suggère que l'utilisation des IPP à visée préventive devrait être proscrite, car elle augmente le risque de pneumopathie d'inhalation chez les patients de réanimation ventilés.

\section{Références}

1. Beaulieu M, Williamson D, Sirois C, Lachaine J (2008) Do proton-pump inhibitors increase the risk for nosocomial pneumonia in a medical intensive care unit? J Crit Care 23(4):513-8

2. Sultan N, Nazareno J, Gregor J (2008) Association between proton pump inhibitors and respiratory infections: a systematic review and meta-analysis of clinical trials. Can J Gastroenterol 22(9):761-6

\section{SP100}

\section{Relation entre la compliance au lavage des mains} et l'incidence des infections liées aux soins dans un service de réanimation médicale marocain : analyse par série temporelle

T. Dendane ${ }^{1}$, V.D Rosenthal ${ }^{2}$, N. Madani ${ }^{3}$, J. Belayachi ${ }^{1}$, K. Abidi ${ }^{1}$, A. Zekraoui ${ }^{4}$, A.A Zeggwagh ${ }^{1}$, R. Abouqal ${ }^{1}$

${ }^{1}$ Service de réanimation médicale, CHU Ibn-Sina, Rabat, Maroc ${ }^{2}$ Bernal Medical Center, Medical College of Buenos Aires and Bernal Medical Center, Buenos Aires, Argentine

${ }^{3}$ Service de réanimation médicale, CHU Ibn-Sina, Rabat, Maroc ${ }^{4}$ Hopital Ibn-Sina, CHU Ibn Sina, Rabat, Maroc

Introduction : L'hygiène des mains est considérée comme étant la mesure la plus importante pour prévenir les infections liées aux soins (ILS). Le but de l'étude était de déterminer la relation temporelle entre le taux d'hygiène des mains et l'incidence des infections liées aux soins en milieu de réanimation médicale.

Matériels et méthodes : L'analyse a été basée sur l'évaluation mensuelle prospective de l'incidence des ILS et du taux de lavage des mains de mai 2007 à septembre 2010. Les ILS étaient définies selon les critères du CDC-NNIS [1]. L'évaluation de la compliance au lavage des mains était réalisée au hasard avant le contact du soignant avec le patient durant le matin, l'après-midi et le soir pour tout le personnel soignant (médecins, infirmiers et aides-soignants). Au moins 120 opportunités de lavage des mains ont été évaluées par mois. La modélisation de l'incidence des ILS a été réalisée par le modèle Autoregressive Integrated Moving-Average (ARIMA) selon la méthode de Box et Jenkins avec fonction de transfert. L'unité statistique était le taux mensuel de l'incidence des ILS, et les variables indépendantes étaient le taux mensuel d'hygiène des mains, l'APACHE II moyen mensuel, l'âge moyen mensuel et le pourcentage du sexe masculin mensuel.

Résultats : Le taux d'incidence mensuel des ILS était en moyenne de 31,9 par 1000 jours par patient (17 à 65,1 ILS/1 000 jours par patient) ; le taux mensuel de lavage des mains était en moyenne de 51,9\% (11 à 77,4 \%). En analyse multivariée, le modèle ARIMA n'a pas montré d'effet de la variation du taux de lavage des mains sur l'incidence des ILS, tous types d'infections confondus. Par contre, il a montré que l'augmentation du taux de lavage des mains était liée à une baisse du taux d'incidence spécifique des pneumonies acquises sous ventilation mécanique avec effet immédiat $(\beta=-4,6 ; p<0,001)$ et effet additionnel retardé à un mois $(\beta=-4,5 ; p<0,001)$, avec $r^{2}=0,69$ (le modèle explique $69 \%$ de la variation du taux d'incidence spécifique). L'augmentation du taux de lavage des mains est également liée à une baisse du taux d'incidence des bactériémies primaires avec effet immédiat $(\beta=-0,1$; $\left.p=0,023 ; r^{2}=0,26\right)$ et à une baisse du taux d'incidence des sepsis cliniques avec effet immédiat $(\beta=-4,4 ; p=0,001)$ et effet additionnel retardé à un mois $\left(\beta=-5,5 ; p=0,05 ; r^{2}=0,56\right)$. Il n'y a pas d'effet de la variation du taux de lavage des mains sur l'incidence des bactériémies associées aux cathéters veineux centraux et sur l'incidence des infections urinaires sur sonde.

Conclusion : Cette étude montre une relation temporelle entre le taux de lavage des mains et l'incidence des pneumonies acquises sous ventilation mécanique, des bactériémies primaires et des sepsis cliniques. Par contre, elle ne montre pas d'association entre le taux de lavage des mains et l'incidence des bactériémies associées aux cathéters veineux centraux, l'incidence des infections urinaires sur sonde et l'incidence des ILS tous types confondus.

\section{Référence}

1. Garner JS, Jarvis WR, Emori TG, et al (1988) CDC definitions for nosocomial infections, 1988. Am J Infect Control 16:128-40

\section{SP101 \\ Évaluation des pratiques concernant la prise en charge des états septiques graves \\ S. Bonnet ${ }^{1}$, D. Honnard ${ }^{1}$, C. Mathurin ${ }^{1}$, A. Pavon ${ }^{2}$, J.-P. Quenot ${ }^{2}$ \\ ${ }^{\prime}$ Service régional d'accueil et d'urgence, CHU de Dijon, hôpital général, Dijon, France \\ ${ }^{2}$ Service de réanimation médicale, $C H U$ de Dijon, complexe du Bocage, Dijon, France}

Introduction : Des recommandations sur la prise en charge précoce des états septiques graves ont été publiées en 2006 dans les suites de la Surviving Sepsis Compaign [1]. L'objectif de notre étude était d'évaluer les pratiques concernant la prise en charge des états septiques graves au service d'accueil et d'urgence (SAU) de Dijon.

Patients et méthodes : Nous avons réalisé une étude monocentrique, rétrospective concernant les patients admis au SAU de Dijon avec un diagnostic de sepsis grave. Les données recueillies concernaient à la fois la prise en charge préhospitalière et la prise en charge au SAU de Dijon.

Résultats : Au total, 99 dossiers ont été étudiés sur la période du 15 octobre 2009 au 15 mars 2010. La moyenne d'âge était de 75,1 ans [72,2-78]. La régulation de ces patients était assurée dans $65 \%$ des cas par le centre 15 . Des comorbidités étaient présentes dans $74 \%$ 
des cas. Le délai moyen d'installation des patients aux urgences était de 1 heure 13 minutes [0 heure 57 minutes- 1 heure 29 minutes] sans différence statistique selon la gravité des patients. Le temps de passage moyen aux urgences des patients était d'environ sept heures avant une mutation. Le temps de passage entre le SAU et la réanimation était en moyenne de 7 heures 28 minutes [ 5 heures 49 minutes -9 heures 7 minutes]. Pour les patients mis en condition aux urgences (voie veineuse centrale, cathéter artériel et/ou intubation orotrachéale), le temps de passage moyen aux urgences était de 8 heures 15 minutes [ 2 heures 57 minutes-13 heures 33 minutes]. Parmi les constantes hémodynamiques relevées, la pression artérielle n'est jamais mentionnée. Nous avons ensuite comparé deux groupes de patients, ceux en sepsis sévère et ceux en choc septique. On trouve une différence significative en ce qui concerne le remplissage vasculaire (respectivement 51 vs $97 \%$, $p<0,001$ ), la prescription des antibiotiques (respectivement 73,6 vs $94 \%, p=0,02$ ), l'appel à un réanimateur (respectivement 13 vs $48 \%$, $p=0,002)$.

Discussion : Les temps d'installation et de passage aux urgences des patients présentant un état septique grave semblent très importants et peuvent donc influencer le pronostic de ces patients. Le remplissage vasculaire, la mise en route d'un traitement antibiotique et l'appel d'un réanimateur sont également des éléments pour lesquels une réflexion est nécessaire pour formaliser une procédure.

Conclusion : L'évaluation des pratiques concernant la prise en charge initiale des patients présentant un état septique grave aux urgences est indispensable, car le pronostic se joue la plupart du temps dans les premières heures.

\section{Référence}

1. Groupe transversal sepsis. Prise en charge initiale des états septiques graves de l'adulte et de l'enfant. www.srlf.org

\section{SP102}

\section{Relation entre défaillances vitales précédant l'infection} acquise aux soins intensifs et gravité de celle-ci H. Maréchal, D. Ledoux, M. Nys, P. Damas

${ }^{1}$ Soins intensifs généraux, centre hospitalier universitaire de Liège, Liège, Belgique

Objectif : La gravité des infections semble liée à la présence de défaillances vitales préalables [1]. Toutes les défaillances jouent-elles un même rôle?

Patients et méthodes : Du $1^{\text {er }}$ janvier 2004 au 31 décembre 2007, le score SOFA [2] a été établi quotidiennement chez 2422 patients hospitalisés pour plus de 48 heures dans les 26 lits de soins intensifs généraux du CHU de Liège. Une dysfonction vitale correspond aux scores 1 et 2 du SOFA, une défaillance aux scores 3 et 4 .

Résultats : Une infection fut diagnostiquée en cours de séjour chez 692 patients, un choc septique chez 201 des patients infectés (29\%). Le Tableau 1 donne le relevé des dysfonctions et défaillances avant toute infection acquise, le pourcentage d'infectés et le pourcentage de chocs septiques parmi les infectés. Toutes les dysfonctions et défaillances s'accompagnent d'une proportion de chocs septiques plus élevée qu'en leur absence, sauf la fonction neurologique qui pourtant accroît clairement le risque infectieux $(p<0,001)$. Le nombre de défaillances non neurologiques précédant l'infection est également lié à la gravité de cette dernière. Plus les patients sont défaillants, plus le risque de choc septique est présent (Tableau 2).

Conclusion : Ces données suggèrent que ce n'est pas tant la réponse de l'hôte qui détermine la gravité d'une infection, mais plutôt la présence de défaillances préalables, excepté la neurologique.

\begin{tabular}{|c|c|c|c|}
\hline \multicolumn{4}{|c|}{ Tableau 1} \\
\hline \multicolumn{4}{|c|}{ Pas de dysfonctions } \\
\hline Fet & Nombre total (\%) & $\%$ Inf & $\%$ choc \\
\hline Resp & $107(4,4)$ & 12,1 & 7,7 \\
\hline Circ & $423(17,5)$ & 29,3 & 12,1 \\
\hline Neur & $1119(46,2)$ & 15,5 & 38,7 \\
\hline Hép & $1468(60,6)$ & 25,0 & 20,7 \\
\hline Plt & $1126(46,6)$ & 28,8 & 21,2 \\
\hline Rénale & $1271(52,5)$ & 26,0 & 17,6 \\
\hline \multicolumn{4}{|c|}{ Dysfonctions } \\
\hline Fet & Nombre total (\%) & $\%$ Inf & $\%$ choc \\
\hline Resp & $1338(55,2)$ & 21,8 & 28,8 \\
\hline Circ & $1154(47,6)$ & 20,6 & 29,0 \\
\hline Neur & $772(31,9)$ & 32,8 & 32,4 \\
\hline Hép & $804(33,2)$ & 33,3 & 34,3 \\
\hline Plt & $1055(43,6)$ & 26,2 & 29,7 \\
\hline Rénale & $676(27,9)$ & 26,3 & 33,7 \\
\hline \multicolumn{4}{|c|}{ Défaillances } \\
\hline Fet & Nombre total (\%) & $\%$ Inf & $\%$ choc \\
\hline Resp & $977(40,3)$ & 39,6 & 30,0 \\
\hline Circ & $845(34,9)$ & 39,1 & 35,5 \\
\hline Neur & $531(21,9)$ & 50,1 & 19,5 \\
\hline Hép & $150(6,2)$ & 38,0 & 57,9 \\
\hline Plt & $239(9,9)$ & 38,1 & 54,9 \\
\hline Rénale & $475(19,6)$ & 38,7 & 45,1 \\
\hline
\end{tabular}

\begin{tabular}{|llll|}
\hline Tableau 2 & & & \\
\hline $\begin{array}{l}\text { Nombre de } \\
\text { défaillances }\end{array}$ & $\begin{array}{l}\text { Nombre total } \\
\text { de patients } \mathbf{\%})\end{array}$ & $\begin{array}{l}\text { Pourcentage } \\
\text { d'infectés }\end{array}$ & $\begin{array}{l}\text { Pourcentage } \\
\text { de choc }\end{array}$ \\
\hline 0 & $961(39,7)$ & 17,9 & 18,6 \\
1 & $706(29,1)$ & 28,5 & 22,4 \\
2 & $449(18,5)$ & 39,6 & 33,1 \\
3 & $181(7,5)$ & 46,4 & 38,1 \\
$>3$ & $125(5,2)$ & 45,6 & 37,9 \\
\hline
\end{tabular}

\section{Références}

1. Damas P, Ledoux D, Nys M, et al (2008) Intensive care unit acquired infections and organ failure. Intensive Care Med 34: 856-64

2. Vincent JL, de Mendonça A, Cantraine F, et al (1998) Use of the SOFA score to assess the incidence of organ dysfunction/failure in intensive care units. Crit Care Med 26:1793-800 